\title{
Polyvinyl Alcohol-Cellulose Acetate Composite Reverses Osmosis Membranes: I. Synthesis and Characterization
}

\author{
Amera Muhammed ${ }^{1 *}$, El-Hashash ${ }^{1}$, Mekewi $^{1}$, Guirguis ${ }^{1}$, Ramadan $^{2}$ and Hassanien ${ }^{1}$ \\ ${ }^{1}$ Chemistry Department, Faculty of Science, Ain-Shams University, Cairo, Egypt \\ ${ }^{2}$ Chemistry Department, Faculty of Science, Helwan University, Cairo, Egypt
}

\begin{abstract}
To overcome the high salinity water resources problem, reverse osmosis $(\mathrm{RO})$ process is considered one of the most required techniques due to its high portable water quality produced. Optimization of membranes quality and function is still pursued and considered as of an important added value to the RO processes. In the present study, thin film PVA/CA+PEG membranes were prepared and the PVA layer was crosslinked by a varying maleic acid concentration at different reaction periods. The polymer composite is to be employed for the reverse osmosis process of brackish, saline and sea water purification. Optimization of reaction conditions and parameters such as salt rejection and water flux that affect on the membrane efficiency were verified. Structural and functional characterization of the PVA/CA composite membranes through Fourier Transform Infrared (FT-IR), thermogravimetric analysis (TGA), X-ray diffraction pattern, scanning electron microscopy (SEM), swelling behavior and membrane mechanical properties were monitored.
\end{abstract}

Keywords: Reverse osmosis membrane; Desalination; Composite film; Polyvinyl alcohol; Cellulose acetate; Polyethylene glycol; Crosslinking agents; Salt rejection.

\section{Introduction}

Membrane and membrane processes were first introduced as an analytical tool in chemical and biomedical laboratories that developed very rapidly into industrial products and methods with significant technical and commercial importance for the separation of many inorganic and organic entities [1-4]. In order to achieve high values of permeability and selectivity, the active layer should be ultrathin and hydrophilic [5-9]. Reverse osmosis membranes are prepared by different techniques, amongst micro porous polymeric membrane, which finds many applications in the fields of dehydration of organic mixtures, water treatment and organic-organic separation [10,11]. A most successful process known as pre-evaporation, the dehydration of aqueous-organic mixtures, well established for the dehydration of isopropanol, acetone, tetrahydrofuran, ethylene glycol and methylene chloride [12-14].

PVA membranes are widely used for dehydration of organics [1518 ] and giving good flux but with a poor selectivity due to the negative impact of membrane swelling. To enhance the membrane performance, a variety of membrane modification techniques are employed such as crosslinking, blending and grafting of several chemical agents to the original polymer matrix $[19,20]$. Among those chemical treatments of membranes, crosslinking has been used extensively to modify membranes physicochemical properties, such as crystallinity, hydrophobicity, and mechanical strength [22-24]. PVA is recognized by its high hydrophilic character, good film-forming properties and outstanding physical and chemical stability, it is then considered as an excellent membrane material for preparation of hydrophilic UF and MF membranes [25].

\section{Materials and Methods}

\section{Chemicals}

Polyvinyl alcohol (PVA) of a molecular weight 133,000 g/mol was supplied by Fisher Scientific. Cellulose acetate (CA) of 39.6\% acetyl content average $(\mathrm{Mw}=50,000)$, polyethylene glycol ( $\mathrm{MW}$ avg. 400), maleic acid and methanol were provided from Aldrich co. Deionized water (DI) was obtained from a Millipore Milli-Q filtration system with a resistivity of $18 \mathrm{~cm}$. solvents and inorganic salts were of reagent grade and used directly without further purification.

\section{Membrane preparation}

Preparation of microporous Poly (vinyl alcohol) support membrane: 1-Poly (vinyl alcohol) casting solution was prepared by dissolving $5 \mathrm{wt} \%$ (PVA) in water at $90^{\circ} \mathrm{C}$ with constant stirring until homogenous solution was obtained.

2- 0.001 wt $\%$ of the maleic acid as crosslinking agent was then added.

3-The polymer solution was then allowed to cast over a glass plate and subsequently placed in an oven at $50^{\circ} \mathrm{C}$ overnight and finally washed with deionized water before further treatment. The effect of maleic acid concentration on the support membrane (PVA) durability was followed through varying its concentration from 0.001 to $0.6 \mathrm{wt} \%$ at reaction periods of 10 to 90 minutes. Figure 1 illustrates the reaction mechanism between PVA and fumaric acid as an analog to that of PVA and maleic acid.

Fabrication of composite membranes (dip coating method): Cellulose acetate was dissolved in methanol at different concentrations of $5,25,40$, and $60 \mathrm{wt} \%$. The solution was deposited coherently onto the top surfaces of different samples of the PVA support membrane.

*Corresponding author: Amera Muhammed, Chemistry Department, Faculty of Science, Ain-Shams University, Cairo, Egypt, E-mail: amera_muhammad@hotmail.com

Received February 22, 2012; Accepted April 21, 2012; Published April 24, 2012

Citation: Muhammed A, Hashash E, Mekewi, Guirguis, Ramadan, et al. (2012) Polyvinyl Alcohol-Cellulose Acetate Composite Reverses Osmosis Membranes: I. Synthesis and Characterization. Hydrol Curr Res 3:131. doi:10.4172/21577587.1000131

Copyright: (c) 2012 Muhammed A, et al. This is an open-access article distributed under the terms of the Creative Commons Attribution License, which permits unrestricted use, distribution, and reproduction in any medium, provided the original author and source are credited. 
PEG was then added to the casting mixture to increase the porosity of the cast membrane while maintaining a high polymer content (15-20\%) and, therefore, sufficiently high viscosity of the casting mixture [26,27]. Graphical representation of the final polymer composite membrane structure after the casting process is shown in figure 2.

The composite membrane environmental location is schematically represented in figure 3.

Membrane characterization and functional features: Structural and functional studies of the PVA/CA grafted RO membranes were conducted and evaluated through the following analytical, functional and performance techniques.

FT-IR structural study: Analysis has been carried out using Infrared spectroscopy, Gensis Unicam FT-IR Spectrophotometer. The dimensions of the window, to which the film was fixed, were $1.5 \times 3$ $\mathrm{cm}$. The polymer membrane was finally chopped and $\mathrm{KBr}$ pellet was prepared. The FT-IR spectrum was investigated in the frequency range $4000-500 \mathrm{~cm}^{-1}$. FT-IR studies were conducted at the Central Laboratory of Ain-Shams University- Abbassia, Cairo.<smiles>CCC(C)O</smiles>

(Fumaric acid, FA )

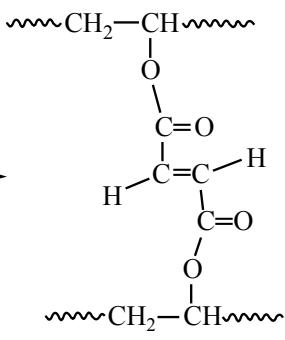

(FA-crosslinked PVA)
Figure 1: Schematic cross-linking mechanism of PVA with fumaric acid.

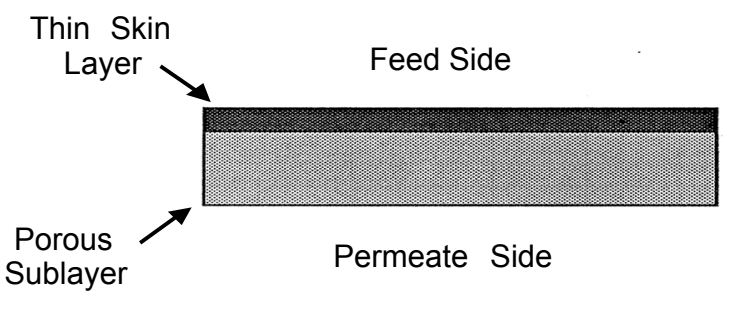

( a )

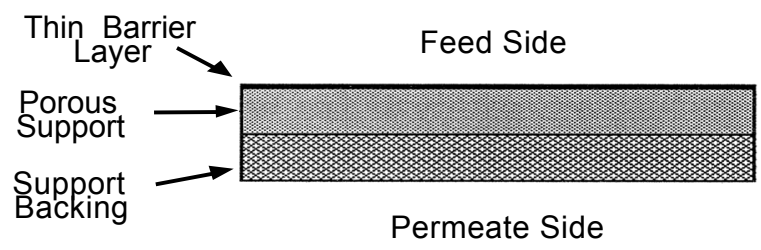

(b)

Figure 2: Schematic of (a) Asymmetric Membrane and (b) Thin-Film Composite Membrane.
Surface and topographical studies of membrane: The surface morphologies of the hydro gels as well as the surface of the membranes were investigated by SEM images using a JSM-5800 instrument, JEOL, (Japan). The polymer films were gold coated before the study. Photographs have been taken X100 magnification. Tapping mode on a commercial AFM (Picoscan, Molecular Imaging, Tempe, AZ, USA) was also employed to aid with topography and phase images of the polymer membrane surfaces. SEM and AFM images were performed at National Center for Radiation Research and Technology, Atomic energy Authority, Egypt.

Mechanical properties: Mechanical measurements were carried out through measuring both tensile strength and elongation percent by using an Instron (Model -1195, England), National Center for Radiation Research and Technology, Atomic energy Authority, Egypt.

Thermal stability profile (TGA): Thermal stability and solid state dehydration changes of membrane samples were carried out using a Shimadzu DT-60H thermal analyzer, Shimadzu, Kyoto, Japan, the measurements were carried out at $500^{\circ} \mathrm{C}$ at a heating rate of $15^{\circ} /$ minute, National Center for Radiation Research and Technology, Atomic energy Authority, Egypt.

G-Reverse Osmosis functional measurements: DDS Reverse

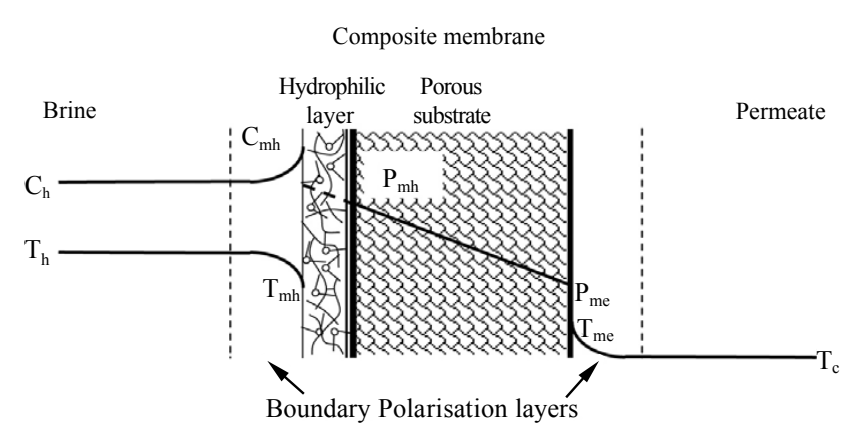

Figure 3: Schematic representation of PVA/CA composite membrane environment.

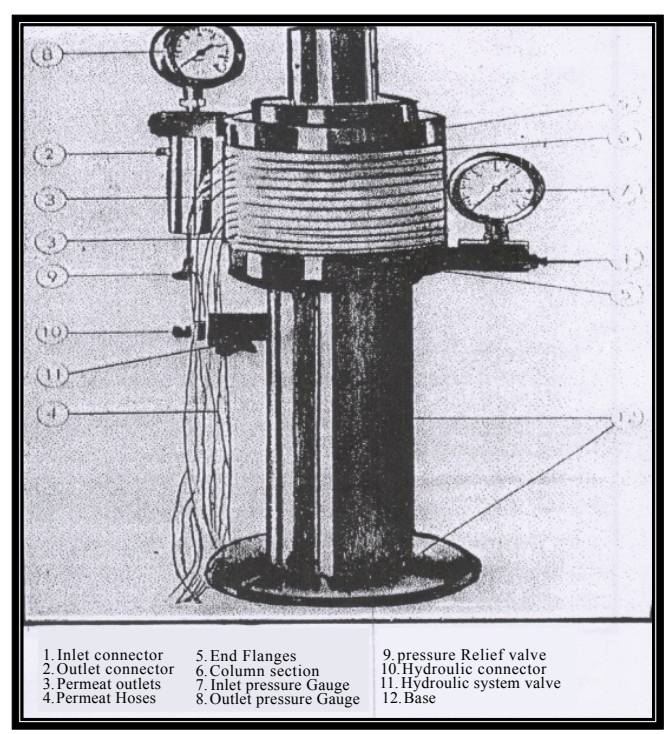

Figure 4: Reverse Osmosis system, model Lab.20. 
Citation: Muhammed A, Hashash E, Mekewi, Guirguis, Ramadan, et al. (2012) Polyvinyl Alcohol-Cellulose Acetate Composite Reverses Osmosis Membranes: I. Synthesis and Characterization. Hydrol Curr Res 3:131. doi:10.4172/2157-7587.1000131

Page 3 of 7

osmosis system available at National Center for Radiation Research and Technology, Atomic energy Authority, Egypt (Figure 4).

\section{Results and Discussion}

\section{PVA, CA, PVA/CA composite membrane structural characterization}

FT-IR structural features: PVA spectral features are well recognized and as shown in figure $5 \mathrm{a}$, where the $-\mathrm{OH}$ familiar groups

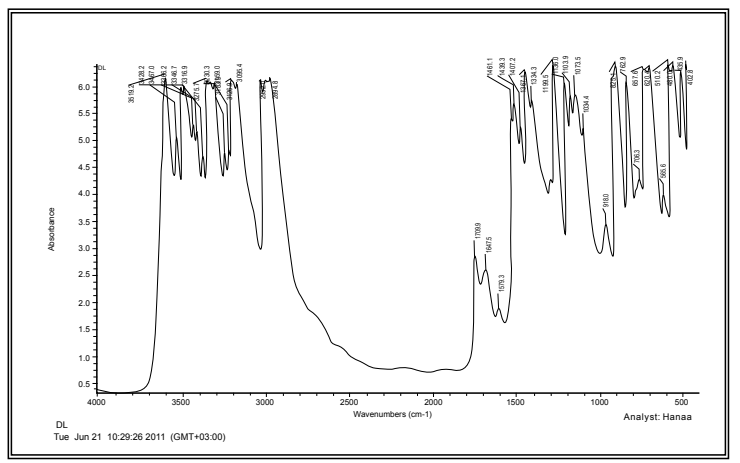

(a)

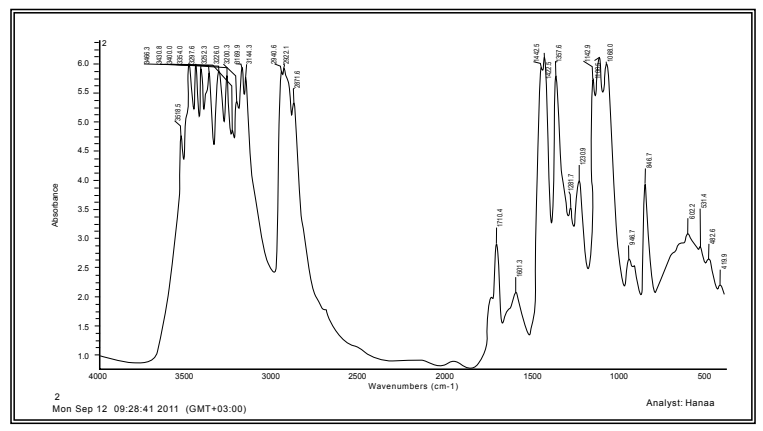

(b)
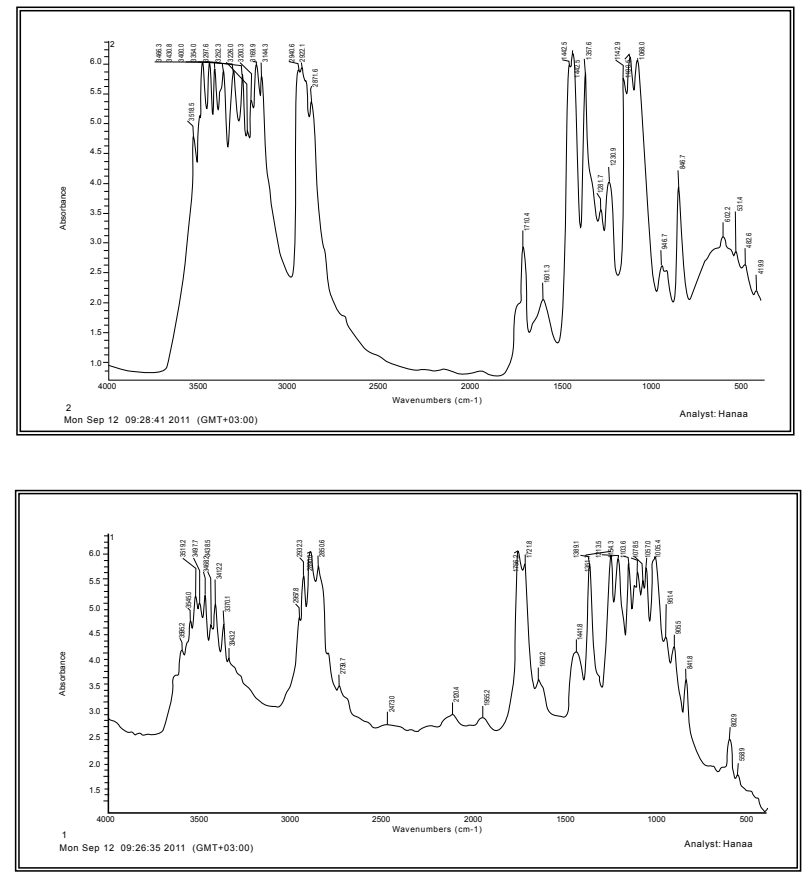

Figure 5: FT-IR of synthetic composite membrane films. are exhibited at the absorption range of $3000-3500 \mathrm{~cm}^{-1}$. When the characteristic $-\mathrm{OH}$ groups of the PVA interchained with the acyl groups of maleic acid, where the stretching vibration at $1647.5 \mathrm{~cm}^{-1}$ is shifted to $1601.3 \mathrm{~cm}^{-1}$ with the prevalence of the $-\mathrm{OH}$ groups and the aliphatic nature of the PVA and the - $\mathrm{CO}$ of the maleic acid in the regions of $1500-1800,1200-1400 \mathrm{~cm}^{-1}$ and $1100 \mathrm{~cm}^{-1}$ where crosslinking effect is affecting not only peaks intensity but also shifting of locations. However, new covalence can be delicately observed but indication of crosslinking action can only be cited at $2871.6 \mathrm{~cm}^{-1}$ and as exhibited when comparing figure $5 \mathrm{a}$ and $5 \mathrm{~b}$.

CA membrane FT-IR spectrum is represented in figure 5c. Vibration bands at $3480 \mathrm{~cm}-1,3043 \mathrm{~cm}-1$ and $1754 \mathrm{~cm}-1$ are exhibited as prominent for the $-\mathrm{OH}, \mathrm{C}-\mathrm{H}$ stretching and $\mathrm{C}=\mathrm{O}$ vibration of ester group. Stretching bands of the alkane groups $\left(\mathrm{CH}_{2}\right.$ and $\left.\mathrm{CH}_{3}\right)$ are shown in the range $1373-1519 \mathrm{~cm}^{-1}$ while bands at $1051-1253 \mathrm{~cm}^{-1}$ correspond to stretching modes of C-O single bond of esters and ethers.

The above FT-IR absorption spectrum of native PVA, crosslinked by maleic acid and of added CA to the mixture indicate on covalency occurrence between the PVA and CA layers which in turn needed to be mutually adhered for durability. Addition of PEG onto the PVA/ CA- maleic acid composite was thought for membrane durability, FTIR spectrum of which is exhibited in figure $5 \mathrm{~d}$. The spectrum exhibits polyethylene glycol absorptions of its primary alcohol groups observed in the region $3378 \mathrm{~cm}^{-1}$. Hence these absorptions, which comprise stretching and bending vibrations restricted to $\mathrm{C}-\mathrm{C}$ stretch, $\mathrm{C}-\mathrm{O}$ stretch, $-\mathrm{CH}$ stretch (methylene absorptions) and the $\mathrm{C}-\mathrm{H}$ bending. The - $\mathrm{OH}$ stretching vibration is also observed in the region $3378 \mathrm{~cm}^{-1}$ exhibiting hydrogen bonded nature. The strong bands shown at 966 $\mathrm{cm}-1$ and $849 \mathrm{~cm}^{-1}$ are indicative to the $\mathrm{C}-\mathrm{C}$ stretching.

The above FT-IR structural features of the native PVA, maleic acid added, CA layered and PEG final addition indicate a composite formed polymer mixture membrane durability of which and functionality will be justified when tested for the purpose of RO desalination.

Scanning electron microscopy (SEM): Revealing the microstructure of the various components of the membrane could be
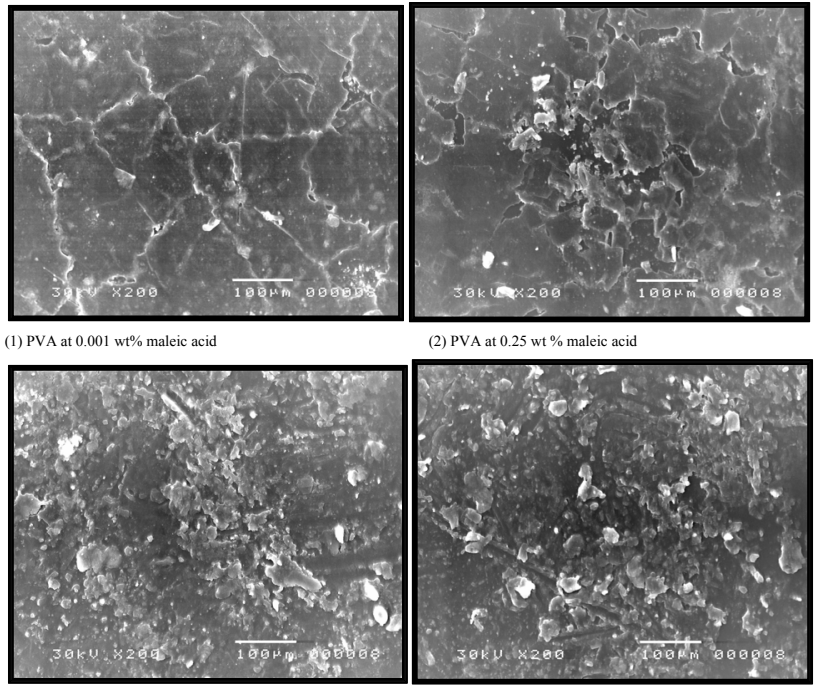

(2) PVA at $0.25 \mathrm{wt} \%$ maleic acid

3) PVA at 0.45 wt\% maleic acid

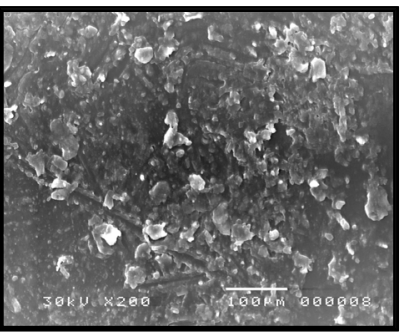

a) Native PVA, b) crosslinked PVA with maleic acid, c) PVA+CA membrane composite, d) $P V A+C A$ \& $P E G$

Figure 6: SEM images of pure PVA at different maleic acid concentration. 
regarded as a useful tool when interpreting durability and function values. Accordingly, figure 6 exhibits the scanning electron micrograph image for PVA-cellulose, PVA/cellulose/PEG materials at different maleic acid concentrations. The SEM images revealed presence of cellulose fiber entanglements on the surface of the membranes. There are slight changes in the surface morphology by addition of maleic acid an increasing porosity due to the increase of the number of pores on the surface. This result evidences that the addition of maleic acid to PVA will increase the performance and efficiency of composite membranes. Mechanical properties are known to be strongly dependent on their morphologies [28], the homogenous morphology could have influenced the tensile properties of the blends obtained in case of PVA \& maleic acid in which the homogenous morphology is accompanied by possessing high tensile and elongation

Figure $7(a, b, c)$ illustrates the SEM images of cellulose membrane before soaking with PVA and after PVA soaking respectively. Thickening of cellulose fibers, figure $8 \mathrm{a}$, are shown to occur after being coated with PVA, figure $8 \mathrm{~b}$ and the inner layers become less visible. Although, some diffused features are visible in the uncoated cellulose membranes due to the presence of some open fibrils, but the fibers are apparently smoothened due to coating of the open fibrils with PVA contrary to PVA membranes, figure 7 , where smooth and featureless surface without pores are noted. Upon crosslinking by PEG to the CA/ PVA material the morphological feature seems to be more compact and as observed from figure $8 \mathrm{c}$. As acetone was used during membrane casting of the $\mathrm{CA}$, it is expected that the molecules are to be immobilized in a glassy state and no pores were formed [29].

Tapping mode atomic force microscopy (AFM): In order
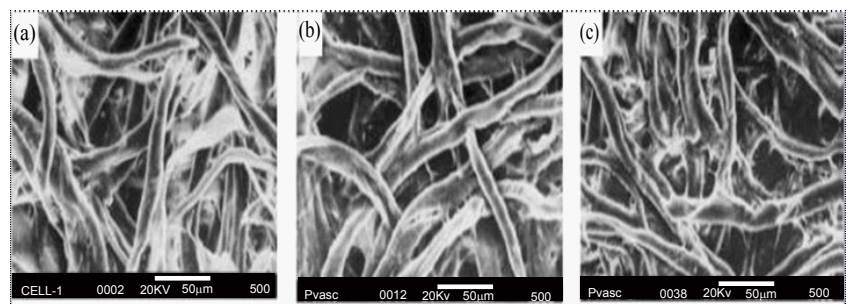

Figure 7: SEM images of (a) pure cellulose acetate, (b) PVA-cellulose composite membranes (c), PVA-CA \& PEG composite membranes.

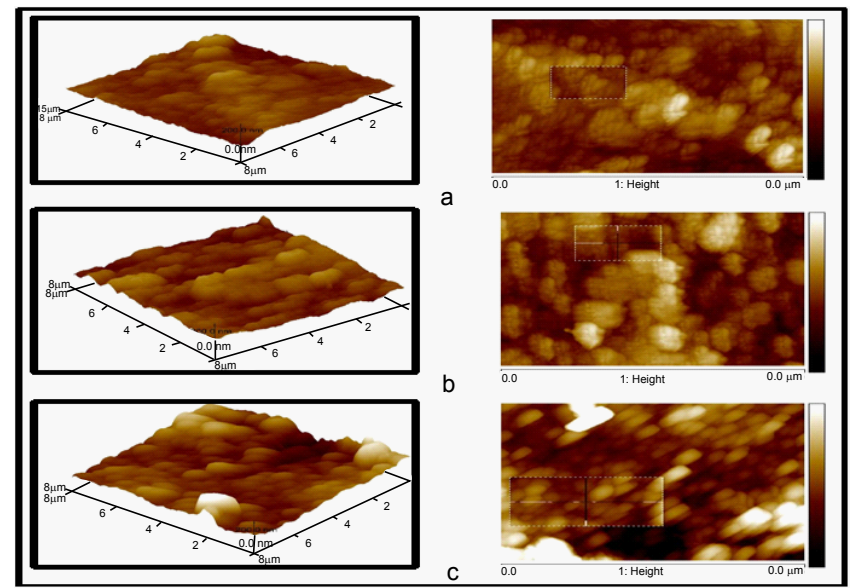

Figure 8: AFM images showing the $2 D$ and $3 D$ surfaces of (a) $C A$, (b) $P V A+C A$ and (c) PVA+CA\&PEG membranes. to investigate clearly the surface morphology of the composite membranes, atomic force microscopy (AFM) was employed, results of which are illustrated in figure 8 which show the images of two and three dimensional scans (scale $8 \mathrm{um}$ ) of the CA, PVA and PVA+CA\&PEG membranes [30]. It could be seen from the images, the membrane without PEG showed nearly a unique and characteristic ridge-andvalley structure consistent [31].

The images demonstrate clearly homogenous morphology and distribution features of CA and PVA, while the PVA+CA \& PEG membrane show many irregularities more than that of PVA, with new protuberances and unevenly distributed features. These irregularities may be due to the insufficient coverage of PEG on membrane surface. As a result, the membrane surface was not completely covered by PEG and the uncovered area showed lower position. Therefore, the roughness parameters of the membrane with PEG increased compared to the membrane without its presence [32].

Thermogravimetric analysis (TGA): The thermal gravimetric analysis (TGA) was performed on the selected polymers evaluated in this study in order to examine the influence of their structural differences on their degradation behavior [33]. Investigation of thermal gravimetric analysis (TGA) of the prepared membranes is presented in figure 9 and is interpreted as follow:

According to figure 9A, i.e. PVA thermal degradation, it is seen that the degradation of all synthetic membranes occurs in three steps:

- The range of first step is from room temperature to $330^{\circ} \mathrm{C}$ in case of PVA/CA membrane. This step represents the evolution of the volatile matter and /or the evaporation of residual absorbed water.

- The second step starts from 330 to $380^{\circ} \mathrm{C}$ which represents the main thermal degradation of the CA chains.

- The third step which takes place over $381^{\circ} \mathrm{C}$ for PVA/CA membrane which symbolizes the carbonization of the degraded products to ash.

Figure 9B, illustrates the degradation of PVA/CA/PEG which takes place also in three steps but is emphasized by more thermal stability than the second composition due may be to addition of PEG to the composition, which the thermal degradation from $402^{\circ} \mathrm{C}$.

Such study proves that the crosslinked PVA/CA membrane by

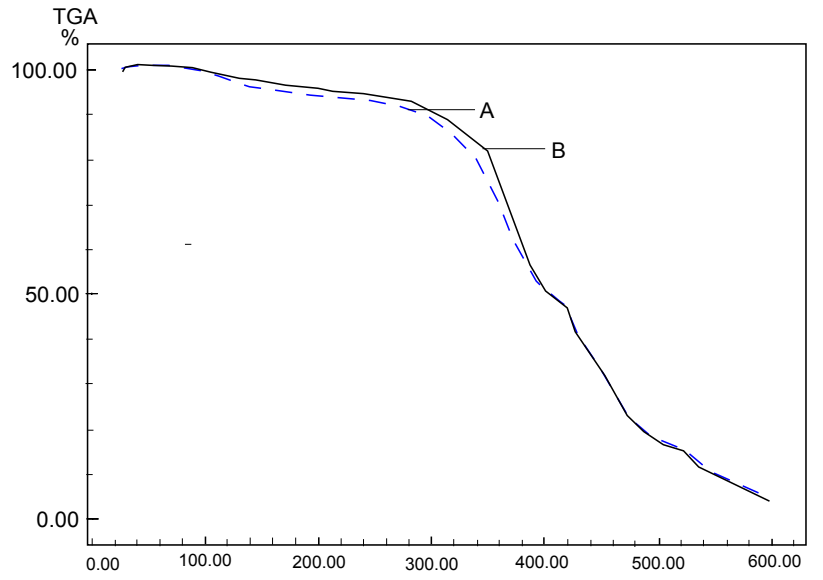

Figure 9: Thermo-gravimetric profile of (A) PVA/CA and (B) PVA/CA/PEG membranes. 


\begin{tabular}{|c|c|c|c|c|}
\hline & $\begin{array}{c}\text { Tensile } \\
\text { Strength }\end{array}$ & & & Elongation \\
\hline Specimen & Yield MPa & Yield \% & Break MPa & Break \% \\
\hline 1 & 34.62 & 11.60 & 13.46 & 34.60 \\
\hline 2 & 38.75 & 18.00 & 58.5 & 49.88 \\
\hline 3 & 63.5 & 21.38 & 20.88 & 137.0 \\
\hline 4 & 64.9 & 72.4 & 75.0 & 269.6 \\
\hline Mean & 40.73 & 33.20 & 25.31 & 84.4 \\
\hline Median & 37.94 & 18.00 & 18.40 & 51.1 \\
\hline Minimum & 18.33 & 10.25 & 6.62 & 26.80 \\
\hline Maximum & 64.9 & 112.0 & 75.0 & 269.6 \\
\hline
\end{tabular}

Table 1: The mechanical properties for some synthetic composite RO membranes.

\begin{tabular}{|c|c|c|c|c|c|c|}
\hline \multirow{2}{*}{$\begin{array}{c}\text { P1 } \\
\text { support }\end{array}$} & \multirow[b]{2}{*}{ P2 } & \multirow[b]{2}{*}{$\begin{array}{l}\mathrm{P} 1 / \mathrm{P} 2 \\
\text { ratio }\end{array}$} & \multicolumn{2}{|c|}{$25 \%$ CA } & \multicolumn{2}{|c|}{$40 \%$ CA } \\
\hline & & & RS (\%) & $\begin{array}{l}\mathrm{JH}_{2} \mathrm{O} \times 10^{-5} \\
\mathrm{gm} / \mathrm{cm} 2 . \mathrm{s}\end{array}$ & RS (\%) & $\begin{array}{l}\mathrm{JH}_{2} \mathrm{O} \times 10^{-5} \\
\mathrm{gm} / \mathrm{cm}^{2} . \mathrm{S}\end{array}$ \\
\hline PVA & CA & $2: 1$ & 30 & 2.74 & 19.78 & 5.38 \\
\hline
\end{tabular}

Table 2: Effect of CA addition on reverse osmosis parameters.

\begin{tabular}{|c|c|c|c|c|c|c|}
\hline \multirow[b]{2}{*}{$\mathbf{P}$} & \multirow[b]{2}{*}{$\mathbf{P}$} & \multirow[b]{2}{*}{ ratio } & \multicolumn{2}{|c|}{ No PEG } & \multicolumn{2}{|c|}{ With $20 \%$ PEG } \\
\hline & & & RS (\%) & $\begin{array}{l}\mathrm{JH}_{2} \mathrm{O} \times 10^{-5} \\
\mathrm{gm} / \mathrm{cm} 2 . \mathrm{s}\end{array}$ & RS (\%) & $\begin{array}{l}\mathrm{JH}_{2} \mathrm{O} \times 10^{-5} \\
\mathrm{gm}^{-5} \mathrm{~cm}^{2} . \mathrm{s}\end{array}$ \\
\hline$C A+P V A$ & PEG & $1: 1$ & 28 & 2.62 & 18.53 & 4.16 \\
\hline
\end{tabular}

Table 3: Effect of $P E G$ addition on reverse osmosis parameters.

PEG has influenced its thermal stability by nearly $20^{\circ} \mathrm{C}[34,35]$.

Mechanical properties: In table (1) shows that the addition of PEG enhanced the resistance of CA and probably reflected the interactions between the free hydroxyl groups of PEG and the chains of CA [36], free $\mathrm{COOH}$ groups which enhance the formation of hydrogen bonding leading to cross-linked network structure. This result leads to an increase of tensile strength with a decrease of the elongation \%.

Effect of CA concentration, PEG additions, solvent effect and other reaction controlling parameters on the composite membrane quality and performance are reported herein.

Effect of cellulose acetate concentration: To improve the properties of the PEG treated PVA/CA membranes, the effect of increasing cellulose acetate (CA) on the water flux and salt rejection parameters, was examined results of which are briefed in table 2. As notified, increasing CA concentration should both the swelling \% and decreasing gelation \% [37]. Accordingly, and as observed from the results, the suitable CA concentration for the purpose of RO desalination process is CA at 25 wt $\%$ of the polymer concentration.

Effect of polyethylene glycol ratio: It was observed that, the suitable PEG concentration is $20 \mathrm{wt} \%$ of the copolymer concentration did improve the water flux and salt rejection parameters, and as recorded, table 3. By addition of PEG, the swelling \% of the obtained membrane increases while the values of both conversion and gelation $\%$ decrease [38].

Selection of solvent type: Choice of the suitable solvent depends upon the solubility of both polymer and monomer and the generation of free radicals in the presence of the solvent $[39,40]$. In case of CA, the effect of many solvents, such as acetone, formic acid and glacial acetic acid was studied. The results have shown that; acetone is the suitable solvent for CA membranes. In case of PVA, indicated that; hot water is the suitable solvent for the preparation of PVA membranes that are homogenous and have good properties.

Effect of cross-linking agent addition: The pervaporation performance of the PVA membrane can be affected by the nature of cross-linking agent used with different dicarboxylic acids, as crosslinking agents. Maleic acid was selected as the PVA's cross-linking agent for the preparation of PVA/CA composite membrane [41]. Accordingly the effect of maleic acid at $0.001 \mathrm{wt} \%$ on the membrane swelling and gelation parameters were studied, results of which are recorded in table 4 .

Effect of reaction temperature and time: It is important to note that the reaction temperature should not exceed $120^{\circ} \mathrm{C}$ (the temperature of membrane formation) $[42,43]$ : otherwise it may affect the polymer deformation, water dehydration. The effect of reaction temperature increase on the swelling ability is illustrated in figure 10 .

The composite membrane preparation of PVA/CA with malice acid as cross-linking was carried out at different reaction times in the range of 45-120 minutes, figure 11 . As can be seen as the reaction time of the membrane preparation is increased, both conversion and water insoluble part \% increase, leading to the formation of condensed network structure that could prevent the penetration of water molecules, and in turn decrease the swelling \%.

Effect of membrane thickness: As shown in table 5, by increasing the membrane thickness, the salt rejection increases while the water flux decreases. This suggests a blocking effect that hinders, to some extent, the movement of water molecules through the membrane [44].

Effect of chemical treatment: Alkaline treatment of the PVA+CA\&PEG membranes (impregnation in $\mathrm{Na}_{2} \mathrm{CO}_{3}$ at room temperature), was carried out to confer ionic character which may result in improving the hydrophilic properties of the grafted membranes. The effect of alkali membrane treatment on the membrane swelling and gelation parameters is offered in table 6. Alkaline treatment of PEG results in converting the free- $\mathrm{COOH}$ groups into the strong electrolyte -COO-Na ${ }^{+}$which possesses higher swelling behaviour due to the broken hydrogen bonding taking place by such chemical conversion of $-\mathrm{COOH}$ into its acrylate $\left(-\mathrm{COO}-\mathrm{Na}^{+}\right)$.

Effect of solvent evaporation: In Tables 7,8 in contrast, low evaporation temperature results in a membrane of high solvent content and the possibility of existence of large pores on the membrane becomes high, this leads to high water flux with low salt rejection [45]. However, the increase in evaporation time at fixed temperature leads to a decrease in water flux with increasing salt rejection.

Effect of feed concentration: In case of salt rejection, the decrease of salt rejection by increasing the feed concentration can be explained by, the passage of salt through the membrane in terms of several

\begin{tabular}{|c|c|c|c|c|c|c|c|c|c|}
\hline \multirow{2}{*}{ Co-polymer } & \multirow{2}{*}{$\mathbf{P}$} & \multicolumn{2}{|c|}{ P/Co-P } & \multicolumn{3}{|c|}{ Untreated } & \multicolumn{3}{|c|}{ Treated with $.001 \%$ maleic acid } \\
\hline & & ratio & $(\%)$ & Swelling ( $\%)$ & Conv (\%) & Gel. (\%) & Swelling (\%) & Conv (\%) & Gel. (\%) \\
\hline \multirow{3}{*}{$C A+P V A$} & \multirow{3}{*}{ PEG } & $2: 1$ & 20 & 49.84 & 81.75 & 68.29 & 110 & 61 & 59.16 \\
\hline & & $4: 1$ & 40 & 52.64 & 79.75 & 60.97 & 120 & 56 & 55 \\
\hline & & $8: 1$ & 80 & 57.39 & 75.22 & 47.32 & 185 & 45 & 45.23 \\
\hline
\end{tabular}

Table 4: Effect of crosslinking on swelling. 


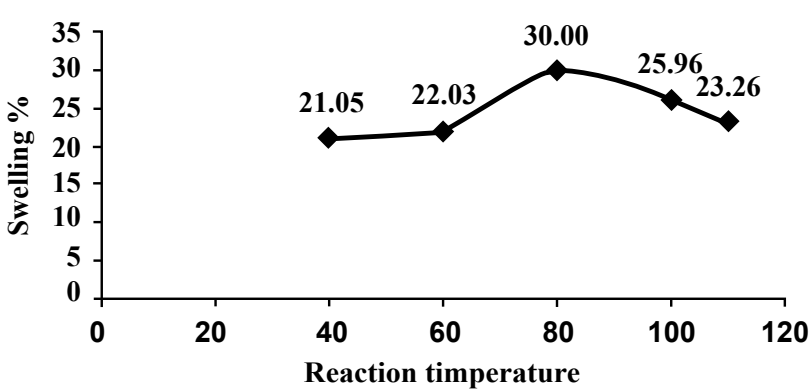

Figure 10: Effect of reaction temperature on swelling, conversion and wate insoluble part (\%) of PVA/CA\&PEG (2:1) crosslinked with malice acid with $0.001 \mathrm{wt} \%$.

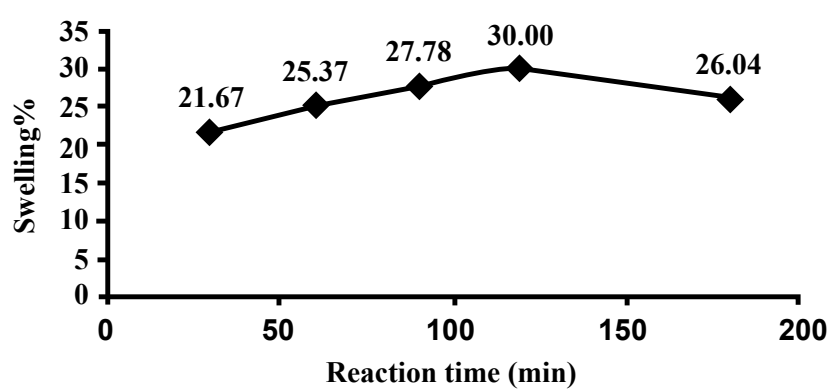

Figure 11: Effect of reaction time on swelling, of PVA/CA\&PEG $(2: 1)$ crosslinked using $0.001 \mathrm{wt} \%$ of malice acid).

\begin{tabular}{|c|c|c|c|}
\hline $\begin{array}{c}\text { Composite } \\
\text { membrane }\end{array}$ & $(\boldsymbol{\mu})$ & $\mathbf{R S}(\%)$ & $\begin{array}{c}\mathbf{J H}_{\mathbf{2}} \mathbf{O x} \mathbf{1 0} \\
\mathbf{9 m} / \mathbf{c m}^{\mathbf{5}} \mathbf{.}\end{array}$ \\
\hline \multirow{3}{*}{ PVA/CA\&PEG } & 60 & 15 & 5.6 \\
\cline { 2 - 4 } & 80 & 18.53 & 4.16 \\
\cline { 2 - 4 } & 190 & 47.53 & 0.77 \\
\hline
\end{tabular}

Table 5: Effect of membrane thickness on reverse osmosis parameters.

\begin{tabular}{|c|c|c|c|c|c|c|c|c|}
\hline \multirow{2}{*}{ Co-P } & \multirow{2}{*}{$\mathbf{P}$} & \multicolumn{3}{|c|}{ Untreated } & \multicolumn{3}{c|}{ Treated } & \\
\cline { 3 - 9 } & & ratio & $\begin{array}{c}\text { Swelling } \\
(\mathbf{\%})\end{array}$ & $\begin{array}{c}\text { Conv } \\
\mathbf{( \% )}\end{array}$ & $\begin{array}{c}\text { Gel. } \\
\text { (\%) }\end{array}$ & $\begin{array}{c}\text { Swelling } \\
(\%)\end{array}$ & Conv(\%) & $\begin{array}{c}\text { Gel. } \\
\text { (\%) }\end{array}$ \\
\hline \multirow{2}{*}{ CA+PVA } & \multirow{2}{*}{ PEG } & $2: 1$ & 49.84 & 81.75 & 68.29 & 110 & 61 & 59.16 \\
\cline { 3 - 9 } & & $4: 1$ & 52.64 & 79.75 & 60.97 & 120 & 56 & 55 \\
\cline { 3 - 9 } & $8: 1$ & 57.39 & 75.22 & 47.32 & 185 & 45 & 45.23 \\
\hline
\end{tabular}

Table 6: Effect of chemical treatment or quaternization on the swelling of some synthetic composite reverse osmosis membranes.

interacting mechanisms including convection, diffusion, and charge repulsion as shown in table 9.

\section{Conclusions}

In the present work interfacial polymerization of CA/PEG thin films over PVA support membranes with different pore structure and chemistry produced polyvinyl alcohol-cellulose acetate composite membranes with widely varying separation performance and interfacial properties suitable as desalination RO membranes. Generally, more large hydrophobic skin layer pores produced more permeable and rough composite membranes due to low \% of polyvinyl alcohol formed within the pores. This produced an overall shorter path length for water and solute transport from the feed side to the permeate side of the polyvinyl alcohol layer. Formation of more polyvinyl alcohol within the pores of relatively porous. Hydrophilic support membranes

\begin{tabular}{|c|c|c|c|c|c|c|c|}
\hline \begin{tabular}{|c|} 
Co- \\
polymer
\end{tabular} & $\mathbf{P}$ & ratio & $(\mu)$ & $\begin{array}{l}\text { Evaporation } \\
\text { Time (min) }\end{array}$ & $\begin{array}{c}\text { Evaporation } \\
\operatorname{Temp}\left({ }^{\circ} \mathrm{C}\right)\end{array}$ & RS (\%) & $\begin{array}{l}\mathrm{JH}_{2} \mathrm{O} \times 10^{-5} \\
\mathrm{gm} / \mathrm{cm}_{2} . \mathrm{s}\end{array}$ \\
\hline \multirow{3}{*}{ CA\&PVA } & \multirow{3}{*}{ PEG } & \multirow{3}{*}{$1: 1$} & \multirow{3}{*}{100} & \multirow{3}{*}{135} & 40 & 18.53 & 4.16 \\
\hline & & & & & 60 & 57.93 & 1.38 \\
\hline & & & & & 80 & 61 & 0.89 \\
\hline
\end{tabular}

Table 7: Effect of evaporation time on reverse osmosis parameters.

\begin{tabular}{|c|c|c|c|c|c|c|c|}
\hline $\begin{array}{c}\text { Co- } \\
\text { polymer }\end{array}$ & $\mathrm{P}$ & ratio & $(\mu)$ & $\begin{array}{l}\text { Evaporation } \\
\text { Time (min) }\end{array}$ & $\begin{array}{l}\text { Evaporation } \\
\text { Temp. }\left({ }^{\circ} \mathrm{C}\right)\end{array}$ & $\begin{array}{l}\text { RS } \\
(\%)\end{array}$ & $\begin{array}{l}\mathrm{JH}_{2} \mathrm{O} \times 10^{-5} \\
\mathrm{gm} / \mathrm{cm}^{2} . \mathrm{s}\end{array}$ \\
\hline \multirow{3}{*}{ CA\&PVA } & \multirow{3}{*}{ PEG } & \multirow{3}{*}{$1: 1$} & \multirow{3}{*}{100} & 135 & \multirow{3}{*}{40} & 18.53 & 4.16 \\
\hline & & & & 170 & & 23.53 & 2.93 \\
\hline & & & & 215 & & 26 & 2.93 \\
\hline
\end{tabular}

Table 8: Effect of evaporation time on reverse osmosis parameters.

\begin{tabular}{|c|c|c|c|c|c|c|}
\hline $\begin{array}{c}\text { Co- } \\
\text { polymer }\end{array}$ & $\mathbf{P}$ & ratio & $\begin{array}{l}\text { TDS } \\
\text { (ppm) }\end{array}$ & RS (\%) & $\begin{array}{c}\text { Salt } \\
\text { Passage } \\
(\%)\end{array}$ & $\begin{array}{c}\mathrm{JH}_{2} \mathrm{O} \\
\times 10^{-5} \mathrm{gm} / \\
\mathrm{cm}^{2} . \mathrm{s}\end{array}$ \\
\hline \multirow{3}{*}{$\mathrm{CA}+\mathrm{PVA}$} & \multirow{3}{*}{ PEG } & \multirow{3}{*}{$1: 1$} & 3333 & 23.65 & 76.35 & 8.02 \\
\hline & & & 42847 & 21.66 & 78.34 & 3.08 \\
\hline & & & 13986.07 & 13.4 & 86.6 & 0.848 \\
\hline
\end{tabular}

Table 9: Effect of feed concentration on reverse osmosis parameters.

produced thinner, less rough polyvinyl alcohol layers with significantly lower permeability.

\section{References}

1. Weatherley LR (1994) Engineering processes for bio-separation. Butterworth Heinemann: Northern Ireland.

2. Barth C, Goncalves MC, Pires ATN, Roeder J, Wolf BA (2000) Asymmetric polysulfone and polyethersulfone membranes: effects of thermodynamic conditions during formation on their performance. J Memb Sci 169: 287-299.

3. M Mulder (1996) Basic principles of membrane technology, Kluwer Academic: The Netherlands.

4. Nakao S (1994) Determination of pore size and pore size distribution. 3 Filtration membranes. J Memb Sci 96: 131-165.

5. Singh S, Khulbe KC, Matsuura T, Ramamurthy P (1998) Membrane characterization by solute transport and atomic force microscopy. J Memb Sci 142: $111-127$.

6. Michaels AS (1980) Analysis and prediction of sieving curves for ultrafiltration membrane: A universal correlation? Sep Sci Technol 15: 1305-1322.

7. Aimar P, Meireles M, Sanchez V (1990) A contribution to the translation of retention curves into pore size distribution for sieving membranes. J Memb Sci 54: 321-338.

8. Narong $P$, James $A E$ (2006) Sodium chloride rejection by a UF ceramic Membrane in relation to its surface electrical properties. Sep and Purif Tech 49: 122-129.

9. Hegazy EA, El-Assy BN, Dessouki MA, Shaker MM (1989) lon-Containing reverse osmosis membranes by radiation grafting method. Radiat Phys Chem 33: 13-18.

10. Huang RYM (1991) Pervaporation Membrane Separation Processes, Elsevier Amsterdam.

11. Oasada Y, Nakagawa T (1992) Membrane Science and Technology, 1st ed Marcel Dekker Inc., New York.

12. Brun JP, Bulvestre G, Kergreis A, Guillou M (1974) Hydrocarbons separation with polymer membranes. I. Butadiene-isobutene separation with nitrile rubber membranes. J Appl Polym Sci 18: 1663-1683.

13. Binning R, Lee R, Jennings J, Martin E (1961) Separation of liquid mixtures by permeation. Ind Eng Chem 53: 45 -50.

14. Gewei Li, Zhang W, Yang J, Wang X (2007) Time-dependence of pervaporation performance for the separation of ethanol/ water mixtures through poly(vinyl alcohol) membrane. J Colloid Interf Sci 306: 337-344. 
Citation: Muhammed A, Hashash E, Mekewi, Guirguis, Ramadan, et al. (2012) Polyvinyl Alcohol-Cellulose Acetate Composite Reverses Osmosis Membranes: I. Synthesis and Characterization. Hydrol Curr Res 3:131. doi:10.4172/2157-7587.1000131

15. Kassotis J, Shmidt J, Hodgins LT, Gregor HP (1985) Modeling of the pore size distribution of ultrafiltration membranes. J Memb Sci 22: 61-76.

16. Chen FR, Chen HF (1996) Pervaporation separation of ethylene glycol-water mixtures using crosslinked PVA-PES composite membranes. Part I. Effects of membrane preparation conditions on pervaporation performances. J Memb Sci 109: $247-256$

17. Huang RYM, Rhim JW (1993) Separation characteristics of pervaporation membrane separation processes using modified poly (vinyl alcohol) membranes. Polym Int 30: 123-128.

18. Kang YS, Lee SW, Kim UY, Shim YS (1990) Pervaporation of water-ethano mixtures through crosslinked and surface-modified poly (vinyl alcohol) membrane. J Memb Sci 51: 215-226.

19. Smitha B, Suhanya D, Sridhar S, Ramakrishna M (2004) Separation of Organic-organic mixtures by pervaporation- a review. J Memb Sci 241: 1-21.

20. Pusch W, Walch A (1982) Membrane structure and its correlation with membrane permeability. J Memb Sci 10: 325-360.

21. Cordone R, Harman WD, Taube H, (1989) .pi.-Heterocyclic complexes of pentaammineosmium(II) and the metal-induced cycloaddition of pyrrole and maleic anhydride. J Am Chem Soc 111: 5969-5970.

22. Guo R, Hu C, Li B, Jiang Z (2007) Pervaporation separation of ethylene glycol/ water mixtures through surface crosslinked PVA membranes: coupling effect and separation performance analysis. J Memb Sci 289: 191-198.

23. Duty RC, Lin HF (1980) Study of the reaction of maleic anhydride with Illinois bituminous coal. Fuel 59: 546-550.

24. Rickard CEF, Quesne PWL (1998) Regio- and Stereoselectivity in Meta Hydride Reduction of the Diels-Alder Adduct of Ergosteryl Acetate and Maleic Anhydride. J Org Chem 63: 1736-1736.

25. Koops GH, Nolten JAM, Mulder MHV, Smolders CA (1994) Selectivity as a function of membrane thickness: gas separation and pervaporation. J Appl Polym Sci 53: 1639-1651.

26. Meier MM, Kanis AL, Soldi V (2004) Characterization and drug Permeation profiles of microporous and dense cellulose acetate membranes: influence of plasticizer and pore formation agent. Inter J of Pharmaceutics 278: 99-110.

27. Rosa DS, Guedes CGF, Casarin F, Braganca FC (2005) The Effect of Mw of $\mathrm{PEG}$ in PCL/CA blends. Polymer Testing 24: 542-548.

28. Kwak SY, Kim SH (2001) Hybrid organic/inorganic reverse osmosis (RO) membrane for bactericidal anti-fouling. 1. Preparation and characterization of $\mathrm{TiO}_{2}$ nanoparticle self-assembled aromatic polyamide thin-film-composite (TFC) Membrane. Environ Sci Techno 35: 2388-2394.

29. Petersen RJ (1993) Composite reverse osmosis and nanofiltration membrane. J Membr Sci 83: 81-150.

30. Kang G, Liu M, Lin B, Cao Y, Yuan Q (2007) A novel method of Surface modification on thin-film composite reverse osmosis membrane by grafting Poly (ethylene glycol). Polymer 48: 1165-1170.
31. Klitzsch E (1984) Berl Geowiss Aabh 50 (A), 23. (2).

32. Arthanareeswaran G, Thanikaivelan P, Srinivasn K, Mohan D, Rajendran M (2004) Synthesis, characterization and thermal studies on cellulose Acetate membranes with additives. Europ Polym J 40: 2153-2159.

33. Lucena MCC, Alencar AEV, Mazzeto SE, Soares SA (2003) The effect of additives on the thermal degradation of cellulose acetate. Polym Degrad Stab 80: 149-155.

34. Qunhui G, Ohya H, Negishi Y (1995) Investigation of the permselectivity of chitosan membrane used in pervaporation separation II. Influences of temperature and membrane thickness. J Memb Sci 98: 223-232.

35. Nijhuis HH, Mulder MHV, Smolders CA (1991) Removal of trace organics from aqueous solutions: effect of membrane thickness. J Memb Sci 61: 99-111.

36. Larsen JW, Quay DM, Roberts JE (1998) Reactions of Pittsburgh No. 8 Coal with Maleic Anhydride. Evidence for the Existence of Reactive Diene Structures in Coal. Energy Fuels 12: 856-863.

37. Rhim JW, Yeom CK, Kim SW (1998) Modification of poly (vinyl alcohol) membranes using sulfur-succinic acid and its application to pervaporation separation of water-alcohol mixtures. J Appl Polym Sci 68: 1717-1723.

38. Yu J, Lee $\mathrm{CH}$, Hong WH (2002) Performances of crosslinked asymmetric poly (vinyl alcohol) membranes for isopropanol dehydration by pervaporation. Chem Eng Process 41: 693-698.

39. Yong SK, Sang WL, Un YK, Jyong SS (1990) Pervaporation of water-ethano mixtures through crosslinked and surface-modified poly (vinyl alcohol) membrane. J Memb Sci 51: 215-226.

40. Nakao S (1994) Determination of pore size and pore size distribution. 3 Filtration membranes. J Memb Sci 96: 131-165.

41. Nam SY, Lee YM (1999) Pervaporation of ethylene glycol-water mixtures: I. Pervaporation performance of surface crosslinked chitosan membranes. J Memb Sci 153: 155-162.

42. Uragami T, Matsuda T, Okuno H, Miyata T (1994) Structure of chemically modified chitosan membranes and their characteristics of permeation and separation of aqueous ethanol solutions. J Memb Sci 88: 243-251.

43. Feng X, Huang RYM (1996) Pervaporation with chitosan membranes I. Separation of water from ethylene glycol by a chitosan/polysulfone composite membrane. J Memb Sci 116: 67-76

44. Mohamed NA, Al-Dossary AOH (2003) Structure-property Relationships for novel wholly aromatic polyamide-hydrazides containing various Proportions of para-phenylene and Meta phenylene units $ш$ Preparation and properties of semi permeable membranes for water desalination by reverse osmosis Separation performance. Euro Polym J 39: 1653-1667.

45. Schaep J, Vandecasteele C, Peeters B, Luyten J, Dotremont C, et al. (1999)

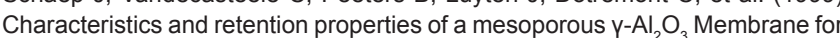
nanofiltration. J Memb Sci 163: 229-237. 